\title{
The generalized Kadanoff-Baym ansatz with initial correlations
}

\author{
Daniel Karlsson, ${ }^{1}$ Robert van Leeuwen, ${ }^{1}$ Enrico Perfetto, ${ }^{2,3}$ and Gianluca Stefanucci ${ }^{3,4}$ \\ ${ }^{1}$ Department of Physics, Nanoscience Center P.O.Box 35 FI-40014 University of Jyväskylä, Finland \\ ${ }^{2}$ CNR-ISM, Division of Ultrafast Processes in Materials (FLASHit), Area della ricerca di Roma 1, Monterotondo Scalo, Italy \\ ${ }^{3}$ Dipartimento di Fisica, Università di Roma Tor Vergata, Via della Ricerca Scientifica, 00133 Rome, Italy \\ ${ }^{4}$ INFN, Sezione di Roma Tor Vergata, Via della Ricerca Scientifica 1, 00133 Roma, Italy
}

(Received 14 June 2018; published 25 September 2018)

\begin{abstract}
Within the nonequilibrium Green's function (NEGF) formalism, the generalized Kadanoff-Baym ansatz (GKBA) has stood out as a computationally cheap method to investigate the dynamics of interacting quantum systems driven out of equilibrium. Current implementations of the NEGF-GKBA, however, suffer from a drawback: real-time simulations require noncorrelated states as initial states. Consequently, initial correlations must be built up through an adiabatic switching of the interaction before turning on any external field, a procedure that can be numerically highly expensive. In this work, we extend the NEGF-GKBA to allow for correlated states as initial states. Our scheme makes it possible to efficiently separate the calculation of the initial state from the real-time simulation, thus paving the way for enlarging the class of systems and external drivings accessible by the already successful NEGF-GKBA. We demonstrate the accuracy of the method and its improved performance in a model donor-acceptor dyad driven out of equilibrium by an external laser pulse.
\end{abstract}

DOI: 10.1103/PhysRevB.98.115148

\section{INTRODUCTION}

The real-time nonequilibrium Green's function (NEGF) technique [1-3] for inhomogeneous systems has received a boost in recent years. One of the reasons is the reinvention of the generalized Kadanoff-Baym ansatz (GKBA) [4] for the solution of the NEGF equations, which has made it possible to perform ab initio simulations of atoms, molecules, and bulk systems thanks to a drastic reduction of the computational effort. The NEGF-GKBA has been used to study, e.g., atoms [5], biologically relevant molecules [6], organic compounds $[7,8]$ as well as a large class of extended systems $[9,10]$ including several two-dimensional layered materials [11,12]. Recently, the scheme has also been used to study model Hamiltonians with Hubbard or extended Hubbard interactions [13-17].

The practical application of the NEGF-GKBA, however, suffers from a drawback. At present it is not known how to include initial correlations in the equations of motion; hence correlations have to be built up in real time. This means taking a noncorrelated state as initial state, evolving the system with an adiabatically switched-on interaction, and then continuing the evolution in the presence of time-dependent external fields if nonequilibrium properties are of interest. The NEGF-GKBA formalism, in the most common approximations, contains a memory kernel that makes the computational effort scale quadratically with the number of time steps. Thus, if we need $N_{\text {ic }}$ time steps to build up initial correlations (using the adiabatic switching) and if the nonequilibrium properties of interest require $N_{\text {prop }}$ more time steps, the overall simulation scales like $\left(N_{\text {ic }}+N_{\text {prop }}\right)^{2}$. Depending on the system, $N_{\text {ic }}$ can be very large, up to the point of making the simulation computationally prohibitive in the physically relevant time window (from $N_{\text {ic }}$ to $N_{\text {ic }}+N_{\text {prop }}$ ). Overcoming this drawback would therefore be of utmost practical value.
We stress from the outset that the reduced computational complexity of NEGF-GKBA with respect to NEGF is currently possible only for many-body self-energies up to the second Born (2B) level, with first- and second-order exchange diagrams evaluated using either the bare Coulomb interaction $v$ or the statically or partially dynamically screened interaction $W$. Indeed, the implementation of, e.g., a full $G W$ or $T$-matrix self-energy would give back the original NEGF scaling in the absence of a GKBA-like expression for the fully dynamically screened interaction $W$ or $T$-matrix $T$. Thus, although NEGFGKBA goes beyond the mean-field approximation and allows for studying systems as diverse as organic molecules and novel 2D materials, an efficient use of NEGF-GKBA for too strongly correlated systems is still out of reach.

In this work, we extend the NEGF-GKBA equation to allow for starting the real-time evolution from an initially correlated (IC) state. This allows for driving the system out of equilibrium already at the beginning of the simulation, thereby reducing the scaling of a calculation from $\left(N_{\text {ic }}+N_{\text {prop }}\right)^{2}$ to $N_{\text {prop. }}^{2}$. The resulting NEGF-GKBA+IC scheme is general and in principle applicable to any system. Existing NEGF-GKBA codes can easily be extended and the additional computational cost is negligible.

The structure of the paper is as follows. We first give a brief introduction to the NEGF formalism and the GKBA. We then discuss the issue of initial correlations and extend the NEGF-GKBA formalism. Two schemes for calculating the initial correlated state are proposed. We present numerical results in a model donor-acceptor complex, show how our method works in practice and demonstrate its accuracy and improved performance with respect to standard NEGF-GKBA simulations. Finally, we conclude and provide an outlook for future directions. 


\section{KADANOFF-BAYM EQUATIONS}

We consider electrons described by the general timedependent second-quantized Hamiltonian in a finite basis

$$
\hat{H}(t)=\sum_{i j \sigma} h_{i j}(t) \hat{c}_{i \sigma}^{\dagger} \hat{c}_{j \sigma}+\frac{1}{2} \sum_{\substack{i j m n \\ \sigma \sigma^{\prime}}} v_{i j m n}(t) \hat{c}_{i \sigma}^{\dagger} \hat{c}_{j \sigma^{\prime}}^{\dagger} \hat{c}_{m \sigma^{\prime}} \hat{c}_{n \sigma} .
$$

The creation (annihilation) operator $\hat{c}_{i \sigma}^{\dagger}\left(\hat{c}_{i \sigma}\right)$ creates (destroys) an electron in basis function $i$ with spin $\sigma$. The single-particle Hamiltonian $h(t)$ contains the kinetic energy as well as a general time-dependent external field. The two-body interaction $v_{i j m n}(t)$ is taken to be time-dependent in order to describe adiabatic switchings or interaction quenches; we do not specify its specific shape further here. Without any loss of generality we assume that the system is in equilibrium for times $t \leqslant 0$. For simplicity, we consider spin-compensated systems, although no complications arise in the more general case.

We describe the nonequilibrium dynamics of the electrons governed by the Hamiltonian in Eq. (1) using NEGF [1-3,18]. The equations of motion for the lesser $\mathcal{G}^{<}$and greater $\mathcal{G}^{>}$ single-particle Green's function are known as the KadanoffBaym equations (KBE) [19] and read (in matrix form)

$$
\begin{aligned}
& {\left[i \vec{\partial}_{t}-h_{\mathrm{HF}}(t)\right] \mathcal{G}^{\lessgtr}\left(t, t^{\prime}\right)} \\
& \quad=\left[\Sigma^{\lessgtr} \cdot \mathcal{G}^{A}+\Sigma^{R} \cdot \mathcal{G}^{\lessgtr}+\Sigma^{\urcorner} \star \mathcal{G}^{\lceil}\right]\left(t, t^{\prime}\right), \\
& \mathcal{G}^{\lessgtr}\left(t, t^{\prime}\right)\left[-i \overleftarrow{\partial}_{t^{\prime}}-h_{\mathrm{HF}}\left(t^{\prime}\right)\right] \\
& \quad=\left[\mathcal{G}^{\lessgtr} \cdot \Sigma^{A}+\mathcal{G}^{R} \cdot \Sigma^{\lessgtr}+\mathcal{G}^{\rceil} \star \Sigma^{\lceil}\right]\left(t, t^{\prime}\right),
\end{aligned}
$$

where we have defined the real-time and imaginary-time convolutions according to

$$
\begin{gathered}
{[A \cdot B]\left(t, t^{\prime}\right) \equiv \int_{0}^{\infty} d \bar{t} A(t, \bar{t}) B\left(\bar{t}, t^{\prime}\right),} \\
{[A \star B]\left(t, t^{\prime}\right) \equiv-i \int_{0}^{\beta} d \bar{\tau} A(t, \bar{\tau}) B\left(\bar{\tau}, t^{\prime}\right),}
\end{gathered}
$$

with $\beta$ the inverse temperature. The imaginary-time convolutions involve the so-called mixed functions with one real time and one imaginary time; they contain information about the IC state [2]. The retarded and advanced functions are defined as

$$
X^{R / A}\left(t, t^{\prime}\right)= \pm \theta\left( \pm\left(t-t^{\prime}\right)\right)\left[X^{>}\left(t, t^{\prime}\right)-X^{<}\left(t, t^{\prime}\right)\right] .
$$

The quantity $\Sigma$ in the KBE is the correlation part of the selfenergy. The time-local mean-field or Hartree-Fock (HF) part of the self-energy is incorporated in $h_{\mathrm{HF}}$, defined as

$$
h_{\mathrm{HF}, i j}(t)=h_{i j}(t)+\sum_{m n} w_{i m n j}(t) \rho_{n m}(t),
$$

where $\rho(t)=-i \mathcal{G}^{<}(t, t)$ is the single-particle density matrix and we have defined $w_{i m n j}(t) \equiv 2 v_{i m n j}(t)-v_{i m j n}(t)$.
In this work, we consider the $2 \mathrm{~B}$ approximation to the correlation self-energy [5]

$$
\Sigma_{i j}^{\lessgtr}(t, \bar{t})=\sum_{m n p q r s} v_{i r p n}(t) w_{m q s j}(\bar{t}) \mathcal{G}_{n m}^{\lessgtr}(t, \bar{t}) \mathcal{G}_{p q}^{\lessgtr}(t, \bar{t}) \mathcal{G}_{s r}^{\gtrless}(\bar{t}, t) .
$$

For future reference, we note that the calculation of the $2 \mathrm{~B}$ self-energy scales like $N_{b}^{5}$ with the number of basis functions $N_{b}$ and that for any fixed $t$ and $\bar{t}$ it does not scale with the number of time steps $N_{t}$.

Knowledge of the lesser/greater Green's functions give access to many observables, e.g., density, current density, spectral function, total energy, etc. Unfortunately, the computational effort to solve the KBE is relatively high since these are integrodifferential equations for two-time functions. Using a time-stepping technique the propagation up to $N_{t}$ time steps scales like $N_{t}^{3}$, provided that the calculation of the self-energy does not scale higher than that [20]. For the most common approximations used in the literature, i.e., the $2 \mathrm{~B}, G W$, and $T$-matrix approximations, the full solution of the KBE does indeed scale cubically with $N_{t}$ [21-24]. This cubic scaling is what prohibits long time evolutions in many systems.

To reduce the computational effort, we reduce the information contained in the unknown functions. Instead of solving the KBE for the Green's function we solve the equation of motion for the single-particle density matrix $\rho(t)$ which is a one-time function. The equation for $\rho$ can be derived from the KBE by subtracting Eq. (3) from Eq. (2), and then letting $t^{\prime} \rightarrow t[2,19]$,

$$
\partial_{t} \rho(t)+i\left[h_{\mathrm{HF}}(t), \rho(t)\right]=-\left(\mathcal{I}(t)+\mathcal{I}^{\text {ic }}(t)+\text { H.c. }\right),
$$

where we have defined the collision integral

$$
\mathcal{I}(t)=\int_{0}^{t} d \bar{t}\left[\Sigma^{>}(t, \bar{t}) \mathcal{G}^{<}(\bar{t}, t)-\Sigma^{<}(t, \bar{t}) \mathcal{G}^{>}(\bar{t}, t)\right]
$$

and the IC integral

$$
\mathcal{I}^{\mathrm{ic}}(t)=-i \int_{0}^{\beta} d \bar{\tau} \Sigma^{\rceil}(t, \bar{\tau}) \mathcal{G}^{\lceil}(\bar{\tau}, t) .
$$

The IC integral $\mathcal{I}^{\text {ic }}(t)$ depends on $t$ only through the integrand, whereas the collision integral $\mathcal{I}(t)$ depends on $t$ through both the integrand and the upper integration limit. Thus the calculation of the right hand side of Eq. (9) scales linearly with the number of time steps $N_{t}$. This implies that the full propagation of the density matrix scales quadratically with $N_{t}$, provided that the calculation of the self-energy does not scale higher than that.

Although the time-stepping technique for $\rho$ is numerically cheaper than for the Green's function, Eq. (9) suffers from a fundamental problem: it is not a closed equation for $\rho$. The collision integral $\mathcal{I}(t)$ involves the off-diagonal (in time) $\mathcal{G}^{\lessgtr}\left(t, t^{\prime}\right)$ and the IC integral contains the mixed functions. In the next section, we discuss the generalized Kadanoff-Baym ansatz (GKBA) to transform $\mathcal{I}$ into a functional of $\rho$, whereas in Sec. IV we present the main result of this work, i.e., a suitable functional form of $\mathcal{I}^{\text {ic }}$ in terms of $\rho$. 


\section{COLLISION INTEGRAL WITH GKBA}

The GKBA [4] is the following ansatz for the lesser and greater Green's function (in matrix form) appearing in the collision integral, Eq. (10),

$$
\begin{aligned}
& \mathcal{G}^{<}\left(t, t^{\prime}\right)=-\left[\mathcal{G}^{R}\left(t, t^{\prime}\right) \rho\left(t^{\prime}\right)-\rho(t) \mathcal{G}^{A}\left(t, t^{\prime}\right)\right], \\
& \mathcal{G}^{>}\left(t, t^{\prime}\right)=\left[\mathcal{G}^{R}\left(t, t^{\prime}\right) \bar{\rho}\left(t^{\prime}\right)-\bar{\rho}(t) \mathcal{G}^{A}\left(t, t^{\prime}\right)\right],
\end{aligned}
$$

where $\bar{\rho}(t) \equiv \hat{\mathbb{1}}-\rho(t)=i \mathcal{G}^{>}(t, t)$. Note that the relation $G^{R}-G^{A}=G^{>}-G^{<}$is guaranteed by this ansatz for any choice of $G^{R}$. Of course, Eq. (12) alone does not transform $\mathcal{I}$ into a functional of the density matrix. We also need to specify the retarded/advanced Green's functions $\mathcal{G}^{R / A}\left(t, t^{\prime}\right)$. These functions satisfy their own KBE and the computational advantage would be lost if we had to solve them numerically. For systems where the average collision time is smaller than the quasiparticle's lifetime the effect of the correlation selfenergy on $\mathcal{G}^{R / A}\left(t, t^{\prime}\right)$ can be discarded, and we can employ the HF approximation to $\mathcal{G}^{R / A}\left(t, t^{\prime}\right)$, i.e.,

$$
\mathcal{G}^{R}\left(t, t^{\prime}\right)=-i \theta\left(t-t^{\prime}\right) \mathcal{T}\left\{e^{-i \int_{t^{\prime}}^{t} h_{\mathrm{HF}}(\bar{t}) d \bar{t}}\right\} .
$$

The symbol $\mathcal{T}\{\}$ denotes chronological time ordering [2].

The calculation of the $\mathrm{HF} \mathcal{G}^{R}\left(t, t^{\prime}\right)$ for all $t^{\prime}<t$ scales linearly in $t$. We mention that there are also other approximations to $\mathcal{G}^{R}\left(t, t^{\prime}\right)$ with the same scaling. They are written in terms of $\rho$ only and contain correlation effects to some extent [16,25-28]. The following discussion applies to these approximations as well.

The expression for the retarded Green's functions, Eq. (13), together with Eq. (12), define the GKBA. Since the HF hamiltonian depends only on $\rho$, see Eq. (7), the right-hand side of Eq. (12) and hence the self-energy of Eq. (8) are functionals of $\rho$. Consequently, the collision integral $\mathcal{I}(t)$, see Eq. (10), becomes a history-dependent functional of $\rho(\bar{t})$ with $\bar{t} \leqslant t$.

\section{INITIAL CORRELATION INTEGRAL WITH GKBA}

\section{A. Drawbacks of a vanishing IC integral}

Without an expression of $\mathcal{I}^{\text {ic }}$ in terms of $\rho$, the equation of motion for the density matrix, Eq. (9), cannot be solved. NEGF-GKBA simulations are usually performed with $\mathcal{I}^{\text {ic }}=$ 0 . However, this is justified only provided that the initial state is noncorrelated. In fact, in the absence of external fields, $\rho(t)=\rho^{\text {eq }}$ should be stationary and consequently $h_{\mathrm{HF}}(t)=$ $h_{\mathrm{HF}}^{\mathrm{eq}}$ is stationary too. If $\mathcal{I}^{\mathrm{ic}}=0$ then Eq. (9) at time $t=0$ implies $\left[\rho^{\mathrm{eq}}, h_{\mathrm{HF}}^{\mathrm{eq}}\right]=0$ since $\mathcal{I}(0)=0$. Therefore $\rho(t)=\rho^{\mathrm{eq}}$ is a solution of Eq. (9) with $\mathcal{I}^{\text {ic }}=0$ only if $\mathcal{I}(t)=0$ for all $t$, i.e., only in the absence of correlations. Vice versa, a correlated density matrix $\rho^{\mathrm{eq}}$ does not commute with $h_{\mathrm{HF}}^{\mathrm{eq}}$ and for it to be stationary in the absence of external fields, $\mathcal{I}^{\text {ic }}$ cannot vanish. This is easily seen by taking again into account that $\mathcal{I}(0)=0$ and hence Eq. (9) at time $t=0$ implies

$$
\mathcal{I}^{\mathrm{ic}}(0)+\text { H.c. }=-i\left[h_{\mathrm{HF}}^{\mathrm{eq}}, \rho^{\mathrm{eq}}\right] .
$$

The common way to circumvent the problem of initially noncorrelated states consists in starting from a noncorrelated $\rho(0)=\rho^{\text {eq }}$ and then build up correlations by a slow switching-on of the interaction. The drawback of this procedure is that the correlation build-up time can be rather long, like in systems with a small gap between the ground state and the lowest excited states. Suppose that we are interested in studying the nonequilibrium dynamics for $N_{\text {prop }}$ time steps and that $N_{\text {ic }}$ time steps are necessary for the IC build-up. The computational effort to perform the $i$ th time step in the physically relevant time-window scales like $N_{\text {ic }}+i$ [since $\mathcal{I}$ in Eq. (10) contains an integral from time step 0 to time step $\left.N_{\text {ic }}+i\right]$ and therefore the cost of the entire simulation scales like $\left(N_{\text {ic }}+N_{\text {prop }}\right)^{2}$.

\section{B. Equivalent expression of the IC integral}

Let us now discuss the removal of the adiabatic switching from the numerical procedure. For this purpose, we inevitably need to find an expression of the IC integral in terms of $\rho$ which satisfies the stationarity property

$$
\mathcal{I}^{\mathrm{ic}}(0)=\mathcal{I}(t)+\mathcal{I}^{\mathrm{ic}}(t)
$$

for any $\rho(t)=\rho^{\text {eq }}$ that is also a solution of the stationary equation (14). The difficulty in deriving such an expression stems from the fact that there is no GKBA-like form for the mixed functions appearing in $\mathcal{I}^{\text {ic }}$, see again Eq. (11).

The solution to the problem is found by rewriting the IC integral in an equivalent manner. In Appendix A, we prove a generalized version of the fluctuation-dissipation theorem and use this generalization in Appendix B to show that the IC integral in Eq. (11) can equivalently be expressed in terms of real-time Green's functions according to [see Eq. (B7)]

$$
\mathcal{I}^{\text {ic }}(t)=\int_{-\infty}^{0} d \bar{t}\left[\Sigma^{>}(t, \bar{t}) \mathcal{G}^{<}(\bar{t}, t)-\Sigma^{<}(t, \bar{t}) \mathcal{G}^{>}(\bar{t}, t)\right]
$$

For $t<0$, when the system is in equilibrium, Eq. (16) follows from the standard fluctuation-dissipation theorems [2] for $\mathcal{G}$ and $\Sigma$. With the generalized fluctuation-dissipation theorem of Appendix A one can show that Eq. (16) is also valid out of equilibrium, i.e., for $t>0$. We emphasize that the equivalence between Eqs. (16) and (11) is an exact result, at zero or finite temperature. For notational convenience, we suppress a convergence factor in Eq. (16), see Eq. (B7).

Let us now employ the GKBA approximation to Eq. (16). The main advantage of Eq. (16) over Eq. (11) is that it contains only lesser and greater Green's functions for which a GKBA exists, and we avoid the necessity of constructing a GKBA for the mixed functions. Therefore Eq. (16) allows us to transform $\mathcal{I}^{\text {ic }}$ into a functional of $\rho$.

While Eq. (16) is an exact relation, it is not obvious that the application of GKBA to Eq. (16) will yield a solution that satisfies the stationarity property. Let us prove that the functional $\mathcal{I}^{\text {ic }}$ indeed fulfills Eq. (15). For any stationary $\rho$ and in the absence of external fields $\mathcal{G}^{R / A}$ is a function of the time difference only, see Eq. (13). Via the GKBA, Eq. (12), the same is true for the lesser and greater Green's functions and hence for the 2B self-energy of Eq. (8). Renaming the integration variable in Eqs. (10) and (16) according to $\bar{t}^{\prime}=$ $\bar{t}-t$ we have that $\mathcal{G} \lessgtr(t, \bar{t})=\mathcal{G} \lessgtr\left(0, \bar{t}^{\prime}\right)$ and hence $\Sigma \lessgtr(t, \bar{t})=$ 
$\Sigma \lessgtr\left(0, \bar{t}^{\prime}\right)$. Using Eqs. (10) and (16), this in turn implies that

$$
\begin{aligned}
\mathcal{I}(t) & +\mathcal{I}^{\mathrm{ic}}(t) \\
= & \int_{-\infty}^{t} d \bar{t}\left[\Sigma^{>}(t, \bar{t}) \mathcal{G}^{<}(\bar{t}, t)-\Sigma^{<}(t, \bar{t}) \mathcal{G}^{>}(\bar{t}, t)\right] \\
& =\int_{-\infty}^{0} d \bar{t}\left[\Sigma^{>}(0, \bar{t}) \mathcal{G}^{<}(\bar{t}, 0)-\Sigma^{<}(0, \bar{t}) \mathcal{G}^{>}(\bar{t}, 0)\right]=\mathcal{I}^{\text {ic }}(0) .
\end{aligned}
$$

Therefore a stationary $\rho^{\text {eq }}$ satisfying Eq. (14) yields a stationary right-hand side in Eq. (9) also for positive times, in the absence of external fields. This demonstrates the formal usefulness of Eq. (16) in the GKBA context. In the next section, we will discuss the practical implications.

\section{Practical implementation of the IC integral with GKBA}

To make the NEGF-GKBA+IC scheme practical we have to perform the IC integral from minus infinity to zero analytically for arbitrary time-dependent drivings switched on at $t>0$. Let us insert the 2B self-energy of Eq. (8) into the expression for $\mathcal{I}^{\text {ic }}$ :

$$
\mathcal{I}^{\text {ic }}(t)=\mathcal{J}^{\text {ic }}(t)-\overline{\mathcal{J}}^{\text {ic }}(t),
$$

where

$$
\begin{aligned}
\mathcal{J}_{i k}^{\mathrm{ic}}(t)= & \sum_{m n p q r s j} v_{i r p n}(t) w_{m q s j} \int_{-\infty}^{0} d \bar{t} \\
& \times \mathcal{G}_{n m}^{>}(t, \bar{t}) \mathcal{G}_{p q}^{>}(t, \bar{t}) \mathcal{G}_{s r}^{<}(\bar{t}, t) \mathcal{G}_{j k}^{<}(\bar{t}, t) e^{\eta \bar{t}},
\end{aligned}
$$

and $\overline{\mathcal{J}}_{i k}^{\text {ic }}(t)$ is defined as in Eq. (18) with the replacement $\mathcal{G}^{\lessgtr} \rightarrow \mathcal{G}^{\gtrless}$. We added the convergence factor $e^{\eta \bar{t}}$ [see Eq. (B7) for details]. In Eq. (18), we took into account that the tensor $w$ is independent of time since we assumed that the Hamiltonian is constant at negative times (for otherwise the system would not be in equilibrium). The contributions $\mathcal{J}^{\text {ic }}$ and $\overline{\mathcal{J}}^{\text {ic }}$ have the same structure; we then discuss $\mathcal{J}^{\text {ic }}$ only. Since $\bar{t}<0<t$, the GKBA of Eq. (12) yields

$$
\begin{aligned}
\mathcal{G}^{>}(t, \bar{t}) & =\mathcal{G}^{R}(t, \bar{t}) \bar{\rho}(\bar{t}), \\
\mathcal{G}^{<}(\bar{t}, t) & =\rho(\bar{t}) \mathcal{G}^{A}(\bar{t}, t) .
\end{aligned}
$$

Furthermore, the retarded/advanced Green's functions in the HF approximation, Eq. (13), satisfies the group property

$$
\begin{aligned}
& \mathcal{G}^{R}(t, \bar{t})=i \mathcal{G}^{R}(t, 0) \mathcal{G}^{R}(0, \bar{t}), \\
& \mathcal{G}^{A}(t, \bar{t})=-i \mathcal{G}^{A}(\bar{t}, 0) \mathcal{G}^{A}(0, t) .
\end{aligned}
$$

Therefore we can rewrite the lesser and greater Green's functions in Eq. (19) as

$$
\begin{aligned}
& \mathcal{G}^{>}(t, \bar{t})=i \mathcal{G}^{R}(t, 0) \mathcal{G}^{>}(0, \bar{t}), \\
& \mathcal{G}^{<}(\bar{t}, t)=-i \mathcal{G}^{<}(\bar{t}, 0) \mathcal{G}^{A}(0, t) .
\end{aligned}
$$

As we shall see below, Eqs. (21) allow for isolating the $t$ dependence in $\mathcal{J}^{\text {ic }}(t)$ as well as for performing the integral over $\bar{t}$ analytically.

To ease the notation we define the time-dependent tensor

$$
\tilde{v}_{i r p n}(t) \equiv \sum_{\tilde{n} \tilde{p} \tilde{r}} v_{i \tilde{r} \tilde{p} \tilde{n}}(t) \mathcal{G}_{\tilde{n} n}^{R}(t, 0) \mathcal{G}_{\tilde{p} p}^{R}(t, 0) \mathcal{G}_{r \tilde{r}}^{A}(0, t) .
$$

We also find it useful to define $\tilde{\mathcal{J}}^{\text {ic }}=\mathcal{J}^{\text {ic }}(t) \mathcal{G}^{R}(t, 0)$ from which we can get back the original $\mathcal{J}^{\text {ic }}(t)$ through $\mathcal{J}^{\text {ic }}(t)=$ $\tilde{\mathcal{J}}^{\text {ic }}(t) \mathcal{G}^{A}(0, t)$ [we have used that $\mathcal{G}^{R}(t, 0) \mathcal{G}^{A}(0, t)=\hat{\mathbb{1}}$ in the HF approximation]. Inserting Eq. (21) into Eq. (18) and taking into account the above definitions, we have

$$
\begin{aligned}
\tilde{\mathcal{J}}_{i k}^{\mathrm{ic}}(t)= & \sum_{m n p q r s j} \tilde{v}_{i r p n}(t) w_{m q s j} \int_{-\infty}^{0} d \bar{t} \mathcal{G}_{n m}^{>}(0, \bar{t}) \\
& \times \mathcal{G}_{p q}^{>}(0, \bar{t}) \mathcal{G}_{s r}^{<}(\bar{t}, 0) \mathcal{G}_{j k}^{<}(\bar{t}, 0) e^{\eta \bar{t}} .
\end{aligned}
$$

As anticipated the $t$ dependence has been isolated since it is now contained only in the tensor $\tilde{v}$. To perform the integral over $\bar{t}$ we observe that $h_{\mathrm{HF}}(\bar{t})=h_{\mathrm{HF}}^{\mathrm{eq}}$ for all $\bar{t}<0$ and therefore

$$
\mathcal{G}^{R}(0, \bar{t})=\left[\mathcal{G}^{A}(\bar{t}, 0)\right]^{\dagger}=-i e^{i h_{\mathrm{HF}}^{\mathrm{eq}} \bar{t}} .
$$

Let us work in the eigenbasis of $h_{\mathrm{HF}}^{\mathrm{eq}}$. In general, this is not the basis resulting from a pure HF calculation since $\rho^{\mathrm{eq}}$ and $h_{\mathrm{HF}}^{\mathrm{eq}}$ do not commute in the correlated case, see again Eq. (14). Denoting by $\epsilon_{n}$ the $n$th eigenvalue of $h_{\mathrm{HF}}^{\mathrm{eq}}$, from Eq. (19), we have

$$
\begin{aligned}
& \mathcal{G}_{n m}^{>}(0, \bar{t})=-i e^{i \epsilon_{n} \bar{t}} \bar{\rho}_{n m}^{\mathrm{eq}}, \\
& \mathcal{G}_{n m}^{<}(\bar{t}, 0)=i \rho_{n m}^{\mathrm{eq}} e^{-i \epsilon_{m} \bar{t}} .
\end{aligned}
$$

Inserting these expressions into Eq. (23) and manipulating $\overline{\mathcal{J}}^{\text {ic }}(t)$ in a similar way we eventually obtain

$$
\mathcal{I}^{\text {ic }}(t)=\tilde{\mathcal{I}}^{\text {ic }}(t) \mathcal{G}^{A}(0, t)
$$

with

$$
\tilde{\mathcal{I}}_{i k}^{\text {ic }}(t)=i \sum_{n p r} \frac{\tilde{v}_{i r p n}(t) \tilde{w}_{n p r k}}{\epsilon_{r}+\epsilon_{k}-\epsilon_{n}-\epsilon_{p}+i \eta},
$$

and the tensor $\tilde{w}$ defined according to

$$
\tilde{w}_{n p r k} \equiv \sum_{m q s j} w_{m q s j}\left(\bar{\rho}_{n m}^{\mathrm{eq}} \bar{\rho}_{p q}^{\mathrm{eq}} \rho_{s r}^{\mathrm{eq}} \rho_{j k}^{\mathrm{eq}}-\rho_{n m}^{\mathrm{eq}} \rho_{p q}^{\mathrm{eq}} \bar{\rho}_{s r}^{\mathrm{eq}} \bar{\rho}_{j k}^{\mathrm{eq}}\right) .
$$

A few remarks are in order. (i) Equations (26) and (27) together with the definitions in Eqs. (22) and (28) allow for including initial correlations in the NEGF-GKBA scheme. The resulting NEGF-GKBA+IC scheme is the main results of this work and it consists in solving Eq. (9) with nonvanishing collision integral and IC integral. The latter is a functional of the initial correlated equilibrium density matrix $\rho^{\mathrm{eq}}$ and of its time-dependent value $\rho(t)$ (through the retarded/advanced Green's functions).

(ii) The Coulomb tensor $v$ and hence $w$ are written in the eigenbasis of $h_{\mathrm{HF}}^{\mathrm{eq}}$. Thus interactions that are sparse in some basis, such as the Hubbard interaction in the site basis, do not necessarily yield a sparse tensor $v$ in the eigenbasis of $h_{\mathrm{HF}}^{\mathrm{eq}}$.

(iii) In the noncorrelated case, $\rho^{\mathrm{eq}}$ is diagonal and it is easy to show that the tensor $\tilde{w}$ is identically zero for $\epsilon_{r}+\epsilon_{k}-$ $\epsilon_{n}-\epsilon_{p}=0$. For a general correlated density matrix $\tilde{w}_{n p r k}$ vanishes whenever $r=n$ and $k=p$ or $r=p$ and $k=n$. We assume the same behavior even for accidental degeneracies and restrict the summation in Eq. (27) to include only those indices for which the denominator is nonvanishing. Thus we can safely set $\eta=0$.

(iv) The extra computational effort for the implementation of the IC integral is minimal. The calculation of $\tilde{w}_{n p r k}$ has 
to be done only once and the summation can be performed efficiently in sequence, scaling at most like $N_{b}^{5}$ where $N_{b}$ is the number of basis functions. The same efficient summation can be used to calculate $\tilde{v}_{n p r k}(t)$ in Eq. (22), although in this case the summation has to be performed for every time step. Having $\tilde{w}$ and $\tilde{v}(t)$ we calculate $\tilde{\mathcal{I}}^{\text {ic }}(t)$ from Eq. (27), another operation that scales like $N_{b}^{5}$. The scaling with the fifth power of $N_{b}$ is the same as that of the summation involved in the 2B self-energy of Eq. (8). Thus $\mathcal{I}(t)$ and $\mathcal{I}^{\text {ic }}(t)$ scale in the same way with the number of basis functions. However, the IC integral does not scale with the number of time steps $N_{t}$ (no time integration) whereas the collision integral scales linearly with $N_{t}$ (integration from time step 0 to time step $N_{t}$ ). Consequently, the inclusion of initial correlations via $\mathcal{I}^{\text {ic }}(t)$ adds a negligible computational cost to standard GKBA simulations. Furthermore, the calculation of $\mathcal{I}^{\text {ic }}(t)$ is completely independent from $\mathcal{I}$ and can be done separately; hence no internal modifications need to be made to an existing GKBA code in order to incorporate initial correlations.

(v) In Appendix C, we show that the above conclusions remain intact when using a given dynamically screened interaction $W\left(t-t^{\prime}\right)$, as that of Refs. [7,29], in place of the bare time-local interaction $v$.

\section{THE EQUILIBRIUM CORRELATED DENSITY MATRIX}

In the NEGF-GKBA+IC scheme the initial and correlated density matrix $\rho^{\mathrm{eq}}$ satisfies Eq. (14), and $\rho(t)=\rho^{\mathrm{eq}}$ is a solution of the equation of motion (9) in the absence of external fields. A scheme to obtain $\rho^{\text {eq }}$ based on solving the equilibrium KBE for the lesser Green's function using the GKBA for the collision integral has recently been proposed in Ref. [30]. In the following, we discuss two alternative methods.

The first method consists in solving Eq. (14) selfconsistently. This equation, however, admits infinitely many solutions since the diagonal of the left and right hand sides vanish in any real basis for Hamiltonians invariant under time-reversal. In fact, Eq. (14) is not a variational equation, rather it is a stationary equation, i.e., it stems from setting $\partial_{t} \rho=0$. The possible solutions do therefore correspond to the infinitely many stationary density matrices of the system. A unique solution can be found by supplementing Eq. (14) with the value of the diagonal occupations $\rho_{n n}=\left\{f_{n}\right\}$ in some basis. To illustrate the self-consistent procedure let us first discuss the noncorrelated case, i.e., $\mathcal{I}^{\text {ic }}=0$. Then, Eq. (14) tells us that $\rho^{\mathrm{eq}}$ is diagonal in the eigenbasis of $h_{\mathrm{HF}}$. We then diagonalize the noninteracting Hamiltonian $h$, find the eigenvectors $\varphi_{n}^{(0)}$, and construct $\rho_{n m}^{(0)}=\delta_{n m} f_{n}$ in the basis of these eigenvectors. In the $(i+1)$-th iteration step we use $\rho^{(i)}$ to calculate $h_{\mathrm{HF}}^{(i)}=h_{\mathrm{HF}}\left[\rho^{(i)}\right]$, find the eigenvectors $\varphi_{n}^{(i+1)}$ and construct $\rho_{n m}^{(i+1)}=\delta_{n m} f_{n}$ in the $(i+1)$-basis. At convergence we have the HF basis with HF occupations $\left\{f_{n}\right\}$. In particular, if $f_{n}=1$ for $n \leqslant N_{\mathrm{el}}$ and zero otherwise the procedure converges to the HF ground state with $2 N_{\mathrm{el}}$ electrons. In the correlated case, the procedure is identical but in the $(i+1)$ th iteration step $\rho_{n m}^{(i+1)}$ is not diagonal. In the eigenbasis $\varphi_{n}^{(i+1)}$ of $h_{\mathrm{HF}}^{(i)}=h_{\mathrm{HF}}\left[\rho^{(i)}\right]$ with eigenvalues $\epsilon_{n}^{(i+1)}$, we have for $n \neq m$,

$$
\rho_{n m}^{(i+1)}=i \frac{\mathcal{I}_{n m}^{\mathrm{ic}}(0)+\mathcal{I}_{m n}^{\mathrm{i} *}(0)}{\epsilon_{n}^{(i+1)}-\epsilon_{m}^{(i+1)}} .
$$

As already observed, this result does not allow to update the diagonal elements. We could either supplement Eq. (29) with $\rho_{n n}^{(i+1)}=f_{n}$ for some reasonable set of occupations or take advantage from a self-consistent Matsubara Green's function calculation providing $\rho_{p q}=\delta_{p q} f_{q}$ in the natural orbital basis $\psi_{q}$ and supplement Eq. (29) with

$$
\rho_{n n}^{(i+1)}=\sum_{q} f_{q}\left|\left\langle\psi_{q} \mid \varphi_{n}^{(i+1)}\right\rangle\right|^{2} .
$$

Independently of the prescription to fix the diagonal elements $\rho_{n n}^{(i+1)}$, at convergence $\rho^{\mathrm{eq}}$ satisfies Eq. (14).

The second method is instead borrowed from standard NEGF-GKBA simulations. We start from an noncorrelated density matrix at time $t=0$ and evolve the system with no external fields in the presence of a slowly increasing interaction $v(t)$ having the property that $v(t<0)=0$ and $v(t>$ $\left.T_{\text {ic }}\right)=v$. The time $T_{\text {ic }}$ is the IC build-up time which should be chosen large enough for $\rho(t)=\rho\left(T_{\text {ic }}\right)$ to be sufficiently stationary when $t$ is larger than $T_{\text {ic }}$. Taking advantage of the fact that $v(t)=0$ for $t \leqslant 0$, the IC integral vanishes at all times $t$ since $\Sigma \lessgtr(t, \bar{t})=0$ for $\bar{t} \leqslant 0$, as can be seen from Eqs. (8) and (16). At the steady state $\rho\left(T_{\text {ic }}\right)=\rho^{\text {eq }}$ satisfies Eq. (14). We emphasize again that the number of time steps for the IC build-up does not affect the computational cost of the subsequent physically relevant time propagation with $\rho(0)=\rho^{\text {eq }}$ as initial state. We also observe that this second method is limited to systems at zero temperature. In fact, due to correlation-induced level crossings and/or splittings of degenerate many-body states, the finite-temperature noninteracting density matrix does not, in general, evolve into the finite-temperature interacting one.

\section{EXAMPLE OF GKBA WITH INITIAL CORRELATIONS}

In this section, we provide numerical evidence that our procedure works and is efficient. As a non-trivial example, we consider the donor-acceptor dyad used in Ref. [16] as a molecular junction to address the ultrafast charge dynamics at the donor-acceptor interface. The system is modelled by a two-levels donor, the levels being the HOMO $(H)$ and LUMO $(L)$, and a linear chain of $N_{a}$ acceptor sites labeled by the site index $a$. The Hamiltonian reads

$$
\begin{aligned}
\hat{H}= & \epsilon_{A} \sum_{a=1}^{N_{a}} \hat{n}_{a}+T_{D A} \sum_{\sigma}\left(\hat{c}_{L \sigma}^{\dagger} \hat{c}_{1 \sigma}+\text { H.c. }\right) \\
& +\sum_{i=H, L} \epsilon_{i} \hat{n}_{i}+T_{A} \sum_{\sigma, a=1}^{N_{a}-1}\left(\hat{c}_{a \sigma}^{\dagger} \hat{c}_{a+1, \sigma}+\text { H.c. }\right) \\
& +U_{D A}(t)\left(\hat{n}_{H}+\hat{n}_{L}-2\right) \sum_{a=1}^{N_{a}} \frac{\hat{n}_{a}-1}{a}
\end{aligned}
$$

where H.c. denotes the hermitian conjugates. We defined $\hat{n}_{i}=$ $\sum_{\sigma} \hat{n}_{i \sigma}$ the occupation of level $i=H, L$ with energy $\epsilon_{i}$ and 

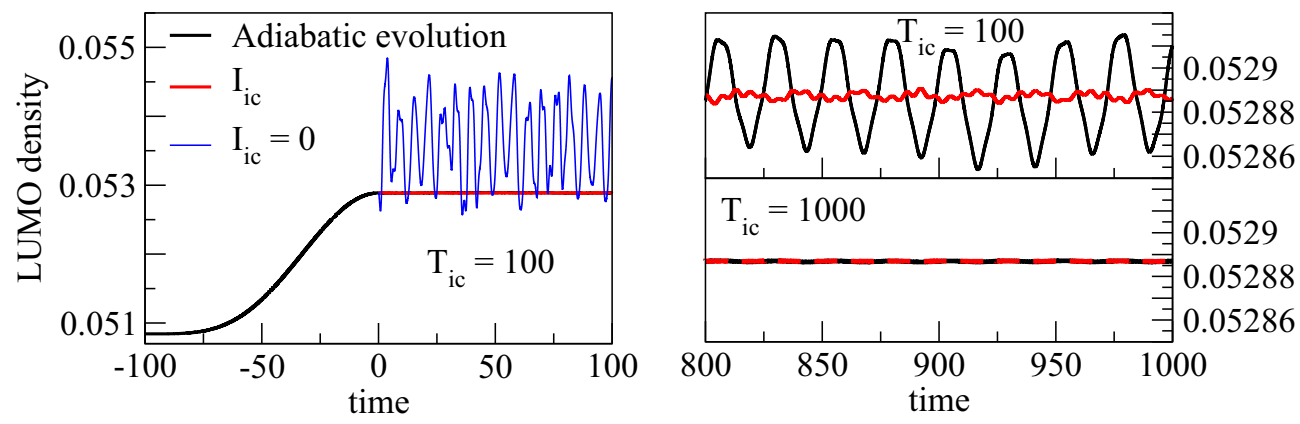

FIG. 1. LUMO occupation, without external fields, for the three types of calculations described in the main text. Total number of time steps is $N_{t}=2 \times 10^{5}$ and the time step is $\Delta t=0.005$. (Left) Evolution using $T_{\mathrm{ic}}=100$ up to $t=100$. Top right panel: long time behavior for $T_{\mathrm{ic}}=100$. (Bottom right) Long time behavior for $T_{\mathrm{ic}}=1000$. In the right panels, we do not show the curve corresponding to calculation (c) $\left[\mathcal{I}_{\text {ic }}(t)=0\right.$, see main text $]$ due to too large oscillations.

likewise for the occupation of the acceptor sites. The system is isolated and the dimension of the single-particle basis is $N_{b}=2+N_{a}$. The LUMO is not coupled to the HOMO but to the first site of the acceptor chain with tunneling amplitude $T_{D A}$. The tunneling amplitude between two nearest neighbor acceptor sites is $T_{A}$. In accordance with Ref. [16], we set the level energies $\epsilon_{H}=-2.92, \epsilon_{L}=-0.92$ and $\epsilon_{A}=-2.08$, and the tunneling amplitudes $T_{D A}=-0.3$ and $T_{A}=-0.2$ (all quantities are in atomic units). The donor-acceptor dyad is half-filled with equal number of up and down electrons. The electrons interact with a density-density type of interaction, and we set the interaction strength $U_{D A}(t)=U_{D A}=0.5$ for positive times.

As time-dependent perturbation we choose

$$
\hat{H}_{\mathrm{ext}}(t)=f(t) \sum_{\sigma}\left(D_{L H} e^{i \Omega t} \hat{c}_{H \sigma}^{\dagger} \hat{c}_{L \sigma}+\text { H.c. }\right)
$$

describing the coupling between a monochromatic electric field of amplitude $f$ and frequency $\Omega$, and the HOMO-LUMO dipole moment $D_{L H}$. We consider a resonant frequency $\Omega=$ $\epsilon_{L}-\epsilon_{H}=2$ and set the value of $D_{H L}=0.3$. The electric field is very strong, $f=1$, and it is active from time $t=$ 0 until time $t=\frac{\pi}{4 D_{L H}} \simeq 2.6$. As we shall see, the external driving transfer one unit of electric charge from the initially filled HOMO to the initially empty LUMO. In all simulations below, we have considered the number of acceptor sites $N_{a}=4$.

\section{A. Simulations without external field}

We first show calculations without external fields to illustrate that the system is stationary with the inclusion of the IC integral. We use the adiabatic switching method to obtain the initially correlated density matrix $\rho^{\text {eq }}$, see Sec. V. The switching protocol was chosen to be

$$
U_{D A}(t)=U_{D A} \times\left\{\begin{array}{ll}
\sin ^{2}\left(\frac{\pi}{2} \frac{t}{T_{\mathrm{ic}}}\right) & t<T_{\mathrm{ic}} \\
1 & t \geqslant T_{\mathrm{ic}}
\end{array} .\right.
$$

We have used the CHEERS code [31] with time step $\Delta t=$ 0.005 to perform three separate calculations: (a) a calculation with $\mathcal{I}_{\mathrm{ic}}(t)=0$ that starts from $t=0$ with the noncorrelated HF density matrix and adiabatically switches on the interac- tion with $T_{\mathrm{ic}}=100$ (for this calculation we have shifted the time axis to set the time origin at $T_{\text {ic }}$ ). (b) A NEGF-GKBA+IC calculation with the IC integral evaluated as described in Sec. IV C that starts from $t=0$ using $\rho(t=0)=\rho^{\mathrm{eq}}$; (c) A calculation with $\mathcal{I}_{\text {ic }}(t)=0$ that starts from $t=0$ using $\rho(t=0)=\rho^{\mathrm{eq}}$. We remind that $\rho^{\mathrm{eq}}=\rho^{\mathrm{eq}}\left(T_{\mathrm{ic}}\right)$ and hence calculations (a) and (b) are expected to coincide for large enough $T_{\text {ic }}$. We also stress that the computational time for calculations (b) and (c) is practically equal.

In Fig. 1, we show the evolution of the LUMO occupation $n_{L}=\rho_{L L}(t)$ up to $t=1000$. From the left panel we conclude that the adiabatic evolution, calculation (a), yields a LUMO occupation that remains stationary after $t>0$, except for small oscillations due to the finiteness of $T_{\text {ic }}$. The same quantity for calculation (b), that includes $\mathcal{I}^{\text {ic }}$, is indeed stationary, even for very long propagation times. Calculation (c), where $\mathcal{I}^{\text {ic }}$ is artificially set to zero, does instead yield a nonstationary $\rho(t)$, as expected from the discussion of the previous section. For long times, the LUMO occupation for both calculations (a) and (b) (top right panel) shows small oscillations due to the finite adiabatic switching time. Increasing the switching time to $T_{\text {ic }}=1000$ the amplitude of the oscillations decreases for both calculations (bottom right panel in Fig. 1). Perhaps remarkably, the correlated density matrix $\rho^{\mathrm{eq}}$ resulting from the adiabatic switching with $T_{\mathrm{ic}}=100$ yields a reasonably stationary $\rho(t)$ in NEGF-GKBA+IC [certainly less oscillatory than that of calculation (a)], indicating that the NEGFGKBA+IC scheme is numerically stable.

\section{B. Simulations with external field}

We now show that also the off-diagonal elements of the density matrix are well-reproduced in NEGF-GKBA+IC. We perform the three types of calculations of the previous section in the presence of the external driving in Eq. (31), and use a very long adiabatic switch-on time $T_{\mathrm{ic}}=1000$ to converge the calculations. The quantities chosen to illustrate the performance of the NEGF-GKBA+IC scheme are the LUMO density, the current $J(t)=2\left|T_{D A}\right| \operatorname{Im}\left[\rho_{L 1}(t)\right]$ flowing through the bond between the LUMO and the first acceptor site and the real part of the off-diagonal HOMO-LUMO matrix element of $\rho(t)$. The results are shown in Fig. 2 up to $t=1000$. 


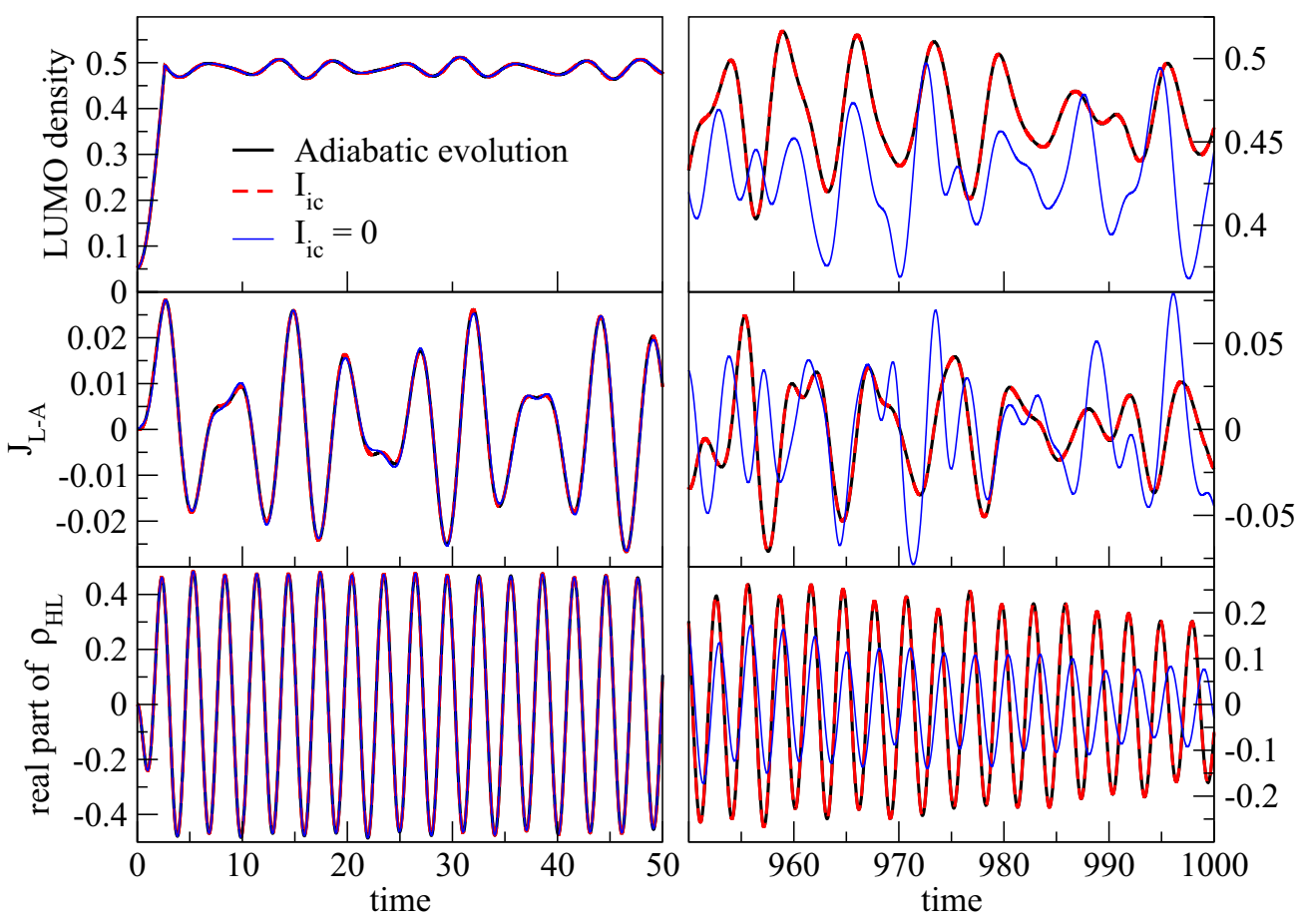

FIG. 2. LUMO occupation (top), current between LUMO and the first acceptor site (middle), and real part of $\rho_{H L}$ (bottom) in the presence of the external driving in Eq. (31) for the three types of calculations described in the main text. Total number of time steps is $N_{t}=2 \times 10^{5}$ and the time step is $\Delta t=0.005$. The quantities are shown in the time range $(0,50)$ (left) and $(950,1000)$ (right).

As anticipated the NEGF-GKBA+IC scheme, calculation (b), correctly reproduces the outcome of standard NEGFGKBA with an adiabatically switched-on interaction, calculation (a). The agreement is excellent all the way to the end of the simulation time. Neglecting the IC integral and starting from the correlated density matrix $\rho^{\mathrm{eq}}$, calculation (c), introduces an error that becomes more severe as the time increases. The general trend is that all quantities can be well-reproduced for short times even without properly accounting for initial correlations, but eventually the agreement tend to deteriorate.

\section{CONCLUSIONS}

Using the NEGF-GKBA+IC scheme we have shown how to separate the calculation of the correlated density matrix from that of the time-dependent responses. By generalizing the fluctuation-dissipation theorem for the Green's function and self-energy, we have derived an equivalent expression of the IC integral suited to be evaluated using the GKBA. With the addition of this IC integral it is possible to use correlated states as initial states, thus removing the bottleneck of a preliminary adiabatic switching. For the most common approximations the computational effort of our method scales favorably and, most importantly, does not slow down an ordinary NEGF-GKBA implementation. Furthermore, the scheme can easily be implemented in any existing GKBA code without internal modifications. The NEGF-GKBA+IC equation widens the class of nonequilibrium phenomena considered so far, allowing for larger systems and/or longer time propagations than was previously feasible. We also emphasize that the proposed scheme is compatible with any technique to obtain the initially correlated density matrix as it does not rely on the adiabatic switching procedure. In fact, the NEGF-GKBA+IC equation is also suitable to study systems at finite temperature (the adiabatic switching procedure is consistent only at zero temperature).

An interesting future prospect is the implementation of many-body approximations to the correlation self-energy that go beyond the ones currently used within the GKBA. We derived a feasible form for the IC integral in the $2 \mathrm{~B}$ approximation, but the fundamental idea is completely general. Indeed, in Appendix C, we provide an expression of the IC integral for the $G W^{\text {eq }}$ approximation, where the dynamically screened interaction is taken from an equilibrium calculation. For other commonly used many-body approximations, like the full $G W$ and $T$-matrix approximation, it is first necessary to find a GKBA-like form of the screened interaction $W$ and $T$ matrix $T$ for otherwise the favourable quadratic scaling with the number of time steps is lost. Perhaps a more immediate direction is the application of the NEGF-GKBA+IC scheme to open systems. This would allow for more efficiently studying, for example, transient quantum transport or photoionization in molecules.

\section{ACKNOWLEDGMENTS}

D.K. acknowledges the Academy of Finland for funding under Project No. 308697, and the Finnish Grid and Cloud Infrastructure for computational resources (urn:nbn:fi:researchinfras-2016072533). G.S. and E.P. acknowledge EC funding through the RISE Co-ExAN (Grant No. GA644076). E.P. also acknowledges funding from the European Union project MaX Materials design at the eXascale H2020-EINFRA-2015-1, Grant Agreement No. 676598 and Nanoscience Foundries and 
Fine Analysis-Europe H2020-INFRAIA-2014-2015, Project No. 654360.

\section{APPENDIX A: GENERALIZED FLUCTUATION-DISSIPATION THEOREM}

The purpose of this appendix is to present a generalized version of the fluctuation-dissipation theorem for the nonequilibrium Green's function, which will be used to derive the equivalence between the two different expressions for the IC integral, Eqs. (11) and (16).

Without any loss of generality, we consider a system in thermal equilibrium at inverse temperature $\beta$ and chemical potential $\mu$ for times $t \leqslant 0$, and out of equilibrium for $t>0$. Let $\hat{\varrho}$ be the many-body thermal density matrix with partition function $\mathcal{Z}$ :

$$
\hat{\varrho}=\frac{e^{-\beta(\hat{H}-\mu \hat{N})}}{\mathcal{Z}}=\sum_{k} \varrho_{k}\left|\psi_{k}\right\rangle\left\langle\psi_{k}\right|,
$$

where $\left|\psi_{k}\right\rangle$ are the many-body eigenstates of $\hat{H}$ with eigenvalue $E_{k}$ and number of particles $N_{k}$. Then,

$$
\varrho_{k}=\frac{e^{-\beta\left(E_{k}-\mu N_{k}\right)}}{\mathcal{Z}} .
$$

By definition, the exact lesser and greater Green's function read [2]

$$
\begin{aligned}
\mathcal{G}_{j i}^{>}\left(t, t^{\prime}\right) & =-i \sum_{k} \varrho_{k}\left\langle\psi_{k}\left|\hat{d}_{j, H}(t) \hat{d}_{i, H}^{\dagger}\left(t^{\prime}\right)\right| \psi_{k}\right\rangle, \\
\mathcal{G}_{j i}^{<}\left(t, t^{\prime}\right) & =i \sum_{k} \varrho_{k}\left\langle\psi_{k}\left|\hat{d}_{i, H}^{\dagger}\left(t^{\prime}\right) \hat{d}_{j, H}(t)\right| \psi_{k}\right\rangle,
\end{aligned}
$$

where the subscript $H$ denotes operators in the Heisenberg picture. Taking into account that the system is in equilibrium for $t^{\prime}<0$ we have

$$
\hat{d}_{i, H}^{(\dagger)}\left(t^{\prime}\right)=e^{i \hat{H} t^{\prime}} \hat{d}_{i}^{(\dagger)} e^{-i \hat{H} t^{\prime}}
$$

where $\hat{d}_{i}^{(\dagger)}$ is the annihilation (creation) operator in the Schrödinger picture. Thus, for $t^{\prime}<0$, the lesser/greater Green's function can be written as

$$
\begin{aligned}
\mathcal{G}_{j i}^{>}\left(t, t^{\prime}\right)= & -i \sum_{k p} \varrho_{k}\left\langle\psi_{k}\left|\hat{d}_{j, H}(t)\right| \psi_{p}\right\rangle \\
& \times\left\langle\psi_{p}\left|\hat{d}_{i}^{\dagger}\right| \psi_{k}\right\rangle e^{i\left(E_{p}-E_{k}\right) t^{\prime}}, \\
\mathcal{G}_{j i}^{<}\left(t, t^{\prime}\right)= & i \sum_{k p} \varrho_{k}\left\langle\psi_{k}\left|\hat{d}_{i}^{\dagger}\right| \psi_{p}\right\rangle \\
& \times\left\langle\psi_{p}\left|\hat{d}_{j, H}(t)\right| \psi_{k}\right\rangle e^{i\left(E_{k}-E_{p}\right) t^{\prime}},
\end{aligned}
$$

where we inserted the completeness relation $\hat{\mathbb{1}}=$ $\sum_{p}\left|\psi_{p}\right\rangle\left\langle\psi_{p}\right|$ between the fermionic operators.

Although the above expressions yield $\mathcal{G} \lessgtr$ only for $t^{\prime}<0$, the right hand sides are well defined for all $t^{\prime}$. We then define the auxiliary lesser and greater Green's functions $\mathcal{G}_{\text {aux } 1}^{\lessgtr}\left(t, t^{\prime}\right)$ as the right hand sides of Eqs. (A6) and (A7) for all $t$ and $t^{\prime}$. Naturally, $\mathcal{G}_{\text {aux } 1}^{\lessgtr}\left(t, t^{\prime}\right)=\mathcal{G} \lessgtr\left(t, t^{\prime}\right)$ only for $t^{\prime}<0$. We now show that these auxiliary functions satisfy a generalization of the fluctuation-dissipation theorem.
Let us consider the Fourier transform of the auxiliary functions:

$$
\mathcal{G}_{\text {aux } 1}^{\lessgtr}\left(t, t^{\prime}\right)=\int \frac{d \omega}{2 \pi} e^{i \omega t^{\prime}} \mathcal{G}_{\text {aux } 1}^{\lessgtr}(t, \omega) .
$$

From Eqs. (A6) and (A7), it is straightforward to get

$$
\begin{aligned}
\mathcal{G}_{\mathrm{aux} 1, j i}^{>}(t, \omega)= & -2 \pi i \sum_{k p} \varrho_{k}\left\langle\psi_{k}\left|\hat{d}_{j, H}(t)\right| \psi_{p}\right\rangle \\
& \times\left\langle\psi_{p}\left|\hat{d}_{i}^{\dagger}\right| \psi_{k}\right\rangle \delta\left(\omega-E_{p}+E_{k}\right), \\
\mathcal{G}_{\text {aux } 1, j i}^{<}(t, \omega)= & 2 \pi i \sum_{k p} \varrho_{k}\left\langle\psi_{k}\left|\hat{d}_{i}^{\dagger}\right| \psi_{p}\right\rangle\left\langle\psi_{p}\right| \\
& \times \hat{d}_{j, H}(t)\left|\psi_{k}\right\rangle \delta\left(\omega-E_{k}+E_{p}\right) .
\end{aligned}
$$

Inserting the obvious relation

$$
\varrho_{k}=e^{-\beta\left(E_{k}-E_{p}\right)+\beta \mu\left(N_{k}-N_{p}\right)} \varrho_{p},
$$

in Eq. (A9), taking into account that only states fulfilling $N_{k}=$ $N_{p}-1$ contribute, and renaming the indices $k \leftrightarrow p$ we obtain

$$
\mathcal{G}_{\text {aux } 1}^{<}(t, \omega)=-e^{-\beta(\omega-\mu)} \mathcal{G}_{\text {aux } 1}^{>}(t, \omega) .
$$

The lesser and greater auxiliary functions can be used to define the retarded and advanced auxiliary functions in the usual manner,

$$
\mathcal{G}_{\text {aux } 1}^{R / A}\left(t, t^{\prime}\right)=\mp i \theta\left(\mp t \pm t^{\prime}\right)\left[\mathcal{G}_{\text {aux } 1}^{>}\left(t, t^{\prime}\right)-\mathcal{G}_{\text {aux } 1}^{<}\left(t, t^{\prime}\right)\right] .
$$

It is straightforward to verify that $\mathcal{G}_{\text {aux1 }}^{>}\left(t, t^{\prime}\right)-\mathcal{G}_{\text {aux }}^{<}\left(t, t^{\prime}\right)=$ $\mathcal{G}_{\text {aux } 1}^{R}\left(t, t^{\prime}\right)-\mathcal{G}_{\text {aux } 1}^{A}\left(t, t^{\prime}\right)$. Fourier transforming the retarded and advanced functions as in Eq. (A8) and taking into account Eq. (A12) we find a generalization of the fluctuationdissipation relations:

$$
\begin{aligned}
& \mathcal{G}_{\text {aux } 1}^{>}(t, \omega)=\bar{f}(\omega-\mu)\left[\mathcal{G}_{\text {aux } 1}^{R}(t, \omega)-\mathcal{G}_{\text {aux } 1}^{A}(t, \omega)\right], \\
& \mathcal{G}_{\text {aux } 1}^{<}(t, \omega)=-f(\omega-\mu)\left[\mathcal{G}_{\text {aux } 1}^{R}(t, \omega)-\mathcal{G}_{\text {aux } 1}^{A}(t, \omega)\right],
\end{aligned}
$$

with Fermi function

$$
f(\omega)=\frac{1}{e^{\beta \omega}+1}, \quad \text { and } \quad \bar{f}(\omega)=1-f(\omega) .
$$

A generalized version of the fluctuation-dissipation relations exists also for $t<0$ and $t^{\prime}$ arbitrary. In this case, from Eqs. (A3) and (A4), we find

$$
\begin{aligned}
\mathcal{G}_{j i}^{>}\left(t, t^{\prime}\right)= & -i \sum_{k p} \varrho_{k}\left\langle\psi_{k}\left|\hat{d}_{j}\right| \psi_{p}\right\rangle\left\langle\psi_{p}\right| \\
& \times \hat{d}_{i, H}^{\dagger}\left(t^{\prime}\right)\left|\psi_{k}\right\rangle e^{i\left(E_{k}-E_{p}\right) t}, \\
\mathcal{G}_{j i}^{<}\left(t, t^{\prime}\right)= & i \sum_{k p} \varrho_{k}\left\langle\psi_{k}\left|\hat{d}_{i, H}^{\dagger}\left(t^{\prime}\right)\right| \psi_{p}\right\rangle \\
& \times\left\langle\psi_{p}\left|\hat{d}_{j}\right| \psi_{k}\right\rangle e^{i\left(E_{p}-E_{k}\right) t} .
\end{aligned}
$$

Again the functions on the right-hand sides are well defined for all $t$ and $t^{\prime}$ and we denote them by $\mathcal{G}_{\text {aux } 2}^{\lessgtr}\left(t, t^{\prime}\right)$. Of course, $\mathcal{G}_{\text {aux } 2}^{\lessgtr}\left(t, t^{\prime}\right)=\mathcal{G}^{\lessgtr}\left(t, t^{\prime}\right)$ only for $t<0$. A derivation similar to the one presented for $\mathcal{G}_{\text {aux } 1}$ can be carried out for this other 
type of auxiliary function leading to

$$
\begin{aligned}
& \mathcal{G}_{\mathrm{aux} 2}^{>}\left(\omega, t^{\prime}\right)=\bar{f}(\omega-\mu)\left[\mathcal{G}_{\mathrm{aux} 2}^{R}\left(\omega, t^{\prime}\right)-\mathcal{G}_{\mathrm{aux} 2}^{A}\left(\omega, t^{\prime}\right)\right], \\
& \mathcal{G}_{\mathrm{aux} 2}^{<}\left(\omega, t^{\prime}\right)=-f(\omega-\mu)\left[\mathcal{G}_{\mathrm{aux} 2}^{R}\left(\omega, t^{\prime}\right)-\mathcal{G}_{\mathrm{aux} 2}^{A}\left(\omega, t^{\prime}\right)\right],
\end{aligned}
$$

where we defined

$$
\mathcal{G}_{\text {aux } 2}^{\lessgtr}\left(t, t^{\prime}\right)=\int \frac{d \omega}{2 \pi} e^{-i \omega t} \mathcal{G}_{\text {aux } 2}^{\lessgtr}\left(\omega, t^{\prime}\right) .
$$

In the derivation of the generalized fluctuation-dissipation relations, Eqs. (A13) and (A17), the only property of the operators $\hat{d}_{j}$ that we have explicitly used is that its action on a state with $N$ particles yields a state with $N-1$ particles. Under the same considerations as in Ref. [2], this leads to a generalized fluctuation-dissipation relation also for the correlation self-energy, since

$$
\begin{aligned}
\Sigma_{j i}^{>}\left(t, t^{\prime}\right) & =-i \operatorname{Tr}\left[\hat{\varrho} \hat{\gamma}_{j, H}(t) \hat{\gamma}_{i, H}^{\dagger}\left(t^{\prime}\right)\right]_{\mathrm{irr}}, \\
\Sigma_{j i}^{<}\left(t, t^{\prime}\right) & =i \operatorname{Tr}\left[\hat{\varrho} \hat{\gamma}_{i, H}^{\dagger}\left(t^{\prime}\right) \hat{\gamma}_{j, H}(t)\right]_{\mathrm{irr}},
\end{aligned}
$$

where the operators $\hat{\gamma}_{i} \equiv \sum_{m n p} v_{\text {imnp }} \hat{d}_{m}^{\dagger} \hat{d}_{n} \hat{d}_{p}$ and the subscript "irr" denotes the irreducible part of the correlator [1,2]. Using the same notation as for the auxiliary Green's functions, we then have

$$
\begin{aligned}
& \Sigma_{\text {aux } 1}^{>}(t, \omega)=\bar{f}(\omega-\mu)\left[\Sigma_{\text {aux } 1}^{R}(t, \omega)-\Sigma_{\text {aux } 1}^{A}(t, \omega)\right], \\
& \Sigma_{\text {aux } 1}^{<}(t, \omega)=-f(\omega-\mu)\left[\Sigma_{\text {aux } 1}^{R}(t, \omega)-\Sigma_{\text {aux } 1}^{A}(t, \omega)\right],
\end{aligned}
$$

and similarly

$$
\begin{aligned}
& \Sigma_{\text {aux2 }}^{>}\left(\omega, t^{\prime}\right)=\bar{f}(\omega-\mu)\left[\Sigma_{\text {aux } 2}^{R}\left(\omega, t^{\prime}\right)-\Sigma_{\text {aux } 2}^{A}\left(\omega, t^{\prime}\right)\right], \\
& \Sigma_{\text {aux } 2}^{<}\left(\omega, t^{\prime}\right)=-f(\omega-\mu)\left[\Sigma_{\text {aux } 2}^{R}\left(\omega, t^{\prime}\right)-\Sigma_{\text {aux } 2}^{A}\left(\omega, t^{\prime}\right)\right] .
\end{aligned}
$$

We emphasize that Eqs. (A13), (A17), (A21), and (A22) are exact relations. In the next appendix, we use them to obtain an equivalent expression of the IC integral.

\section{APPENDIX B: IC INTEGRAL IN TERMS OF REAL-TIME LESSER AND GREATER GREEN'S FUNCTIONS}

We consider the time-off-diagonal generalization of the IC integral in Eq. (11):

$$
I^{\mathrm{ic}}\left(t_{1}, t_{2}\right)=-i \int_{0}^{\beta} d \tau \Sigma^{\rceil}\left(t_{1}, \tau\right) \mathcal{G}^{\lceil}\left(\tau, t_{2}\right) .
$$

When setting $t_{1}=t_{2}=t>0$, we obtain the original IC integral, i.e., $\mathcal{I}^{\text {ic }}(t)=I^{\text {ic }}(t, t)$. To make use of the generalized fluctuation-dissipation theorems in Eq. (B1), we rewrite $\Sigma^{\urcorner}$ and $\mathcal{G}^{\Gamma}$ in terms of $\Sigma^{<}$and $\mathcal{G}^{>}$, respectively. This rewriting can be done by the same considerations as in Ref. [2], and reads

$$
\begin{aligned}
\mathcal{G}^{\Gamma}\left(\tau, t_{2}\right) & =e^{\mu \tau} \mathcal{G}_{\text {aux } 2}^{>}\left(-i \tau, t_{2}\right), \\
\Sigma^{\rceil}\left(t_{1}, \tau\right) & =e^{-\mu \tau} \Sigma_{\text {aux } 1}^{<}\left(t_{1},-i \tau\right),
\end{aligned}
$$

where the auxiliary lesser and greater functions for complex times are defined via the analytic continuation $t \rightarrow-i \tau$ in Eqs. (A15) and (A20). The factors containing $\mu$ in the above equations will cancel when inserted into Eq. (B1), yielding

$$
I^{\mathrm{ic}}\left(t_{1}, t_{2}\right)=-i \int_{0}^{\beta} d \tau \Sigma_{\text {aux } 1}^{<}\left(t_{1},-i \tau\right) \mathcal{G}_{\text {aux } 2}^{>}\left(-i \tau, t_{2}\right) .
$$

Furthermore, the Fourier transform of the auxiliary quantities, Eqs. (A8) and (A18), yields

$$
\begin{aligned}
& \mathcal{G}_{\text {aux } 2}^{>}\left(-i \tau, t_{2}\right)=\int \frac{d \omega_{2}}{2 \pi} e^{-i \omega_{2}(-i \tau)} \mathcal{G}_{\text {aux } 2}^{>}\left(\omega_{2}, t_{2}\right) \\
& \Sigma_{\text {aux } 1}^{<}\left(t_{1},-i \tau\right)=\int \frac{d \omega_{1}}{2 \pi} e^{i \omega_{1}(-i \tau)} \Sigma_{\text {aux } 1}^{<}\left(t_{1}, \omega_{1}\right) .
\end{aligned}
$$

Inserting these relations into Eq. (B2) and integrating over $\tau$ yields

$$
\begin{aligned}
I^{\mathrm{ic}}\left(t_{1}, t_{2}\right)= & -i \int \frac{d \omega_{1} d \omega_{2}}{4 \pi^{2}} \frac{e^{\beta\left(\omega_{1}-\omega_{2}\right)}-1}{\omega_{1}-\omega_{2}-i \eta} \\
& \times \Sigma_{\text {aux } 1}^{<}\left(t_{1}, \omega_{1}\right) \mathcal{G}_{\text {aux } 2}^{>}\left(\omega_{2}, t_{2}\right),
\end{aligned}
$$

where we, in the denominator, added to $\omega_{1}$ a small negative imaginary part $\omega_{1} \rightarrow \omega_{1}-i \eta / 2$, and likewise $\omega_{2} \rightarrow \omega_{2}+$ $i \eta / 2$ to regularize the integral (the limit $\eta \rightarrow 0$ should be taken at the end of the calculation [2]).

We now use the generalized fluctuation-dissipation relations for $\Sigma_{\text {aux } 1}^{<}$and $\mathcal{G}_{\text {aux2 }}^{>}$which, together with the relation

$$
\begin{aligned}
& f\left(\omega_{1}-\mu\right) \bar{f}\left(\omega_{2}-\mu\right)\left(e^{\beta\left(\omega_{1}-\omega_{2}\right)}-1\right) \\
& \quad=f\left(\omega_{2}-\mu\right)-f\left(\omega_{1}-\mu\right),
\end{aligned}
$$

yields

$$
I^{\mathrm{ic}}\left(t_{1}, t_{2}\right)=-i \int \frac{d \omega_{1} d \omega_{2}}{4 \pi^{2}} \frac{\left[\Sigma_{\text {aux } 1}^{R}\left(t_{1}, \omega_{1}\right)-\Sigma_{\text {aux } 1}^{A}\left(t_{1}, \omega_{1}\right)\right] \mathcal{G}_{\text {aux } 2}^{<}\left(\omega_{2}, t_{2}\right)-\Sigma_{\text {aux } 1}^{<}\left(t_{1}, \omega_{1}\right)\left[\mathcal{G}_{\text {aux } 2}^{R}\left(\omega_{2}, t_{2}\right)-\mathcal{G}_{\text {aux } 2}^{A}\left(\omega_{2}, t_{2}\right)\right]}{\omega_{1}-\omega_{2}-i \eta} .
$$

Writing the denominator as

$$
\frac{1}{i\left(\omega_{1}-\omega_{2}-i \eta\right)}=\int_{-\infty}^{0} d \bar{t} e^{i\left(\omega_{1}-\omega_{2}-i \eta\right) \bar{t}}
$$


and recognizing the inverse Fourier transform of the quantities under the integral sign in Eq. (B4) we can write

$$
I^{\mathrm{ic}}\left(t_{1}, t_{2}\right)=\int_{-\infty}^{0} d \bar{t}\left[\left(\Sigma_{\text {aux } 1}^{R}\left(t_{1}, \bar{t}\right)-\Sigma_{\text {aux } 1}^{A}\left(t_{1}, \bar{t}\right)\right) \mathcal{G}_{\text {aux } 2}^{<}\left(\bar{t}, t_{2}\right)-\Sigma_{\text {aux } 1}^{<}\left(t_{1}, \bar{t}\right)\left(\mathcal{G}_{\text {aux } 2}^{R}\left(\bar{t}, t_{2}\right)-\mathcal{G}_{\text {aux } 2}^{A}\left(\bar{t}, t_{2}\right)\right)\right] e^{\eta \bar{t}}
$$

Next, we observe that for $\bar{t}<0$ the first auxiliary self-energy $\Sigma_{\text {aux } 1}$ is identical to the self-energy $\Sigma$ and the second auxiliary Green's function $\mathcal{G}_{\text {aux } 2}$ is identical to the Green's function $\mathcal{G}$. Furthermore, using $\mathcal{G}^{>}-\mathcal{G}^{<}=\mathcal{G}^{R}-\mathcal{G}^{A}$, and likewise for $\Sigma$, we find

$$
\begin{aligned}
I^{\mathrm{ic}}\left(t_{1}, t_{2}\right)= & \int_{-\infty}^{0} d \bar{t}\left[\Sigma^{>}\left(t_{1}, \bar{t}\right) \mathcal{G}^{<}\left(\bar{t}, t_{2}\right)\right. \\
& \left.-\Sigma^{<}\left(t_{1}, \bar{t}\right) \mathcal{G}^{>}\left(\bar{t}, t_{2}\right)\right] e^{\eta \bar{t}} .
\end{aligned}
$$

If we now let $t_{2} \rightarrow t_{1}=t>0$, we obtain the sought-after equivalence between the two alternative forms of the IC integral, i.e., Eqs. (11) and (16).

\section{APPENDIX C: INITIAL CORRELATIONS FOR THE $G W^{\text {eq }}$ APPROXIMATION}

Let us consider the $G W$ approximation to the lesser and greater self-energy

$$
\Sigma_{i j}^{\lessgtr}(t, \bar{t})=i \mathcal{G}_{i j}^{\lessgtr}(t, \bar{t}) W_{i j}^{\lessgtr}(t, \bar{t}),
$$

and let us insert the relation Eq. (21) in Eq. (C1). The first term $\mathcal{J}_{i k}^{\text {ic }}(t)$ of the IC integral in Eq. (16) reads

$$
\begin{aligned}
\mathcal{J}_{i k}^{\mathrm{ic}}(t)= & i \sum_{m n p} \mathcal{G}_{i n}^{R}(0, t)\left[\int_{-\infty}^{0} d \bar{t} \mathcal{G}_{n m}^{>}(0, \bar{t}) W_{i m}^{>}(t, \bar{t})\right. \\
& \left.\times \mathcal{G}_{m p}^{<}(\bar{t}, 0)\right] \mathcal{G}_{p k}^{A}(0, t) .
\end{aligned}
$$

In a full $G W$ calculation, the screened interaction $W$ is a functional of the nonequilibrium $\mathcal{G}$. In this case, the use of the GKBA does not reduce the cubic scaling with the number of time steps of the KBE.

To introduce dynamical screening to some extent we consider a prescribed screened interaction like, e.g., the one resulting from an equilibrium calculation $W=W^{\mathrm{eq}}$. This is the case in $G W_{0}$, where $W_{0}$ is obtained from the RPA response function with bare or HF equilibrium Green's function. Another example is the $W$ of Refs. [7,29] where the response function satisfies the Bethe-Salpeter equation with a frequency-dependent kernel. The advantage of working with an equilibrium screened interaction is that $W^{\mathrm{eq}}(t, \bar{t})$ depends only on $t-\bar{t}$. Fourier transforming according to

$$
W^{\mathrm{eq}, \lessgtr}(t, \bar{t})=\int \frac{d \omega}{2 \pi} e^{-i \omega(t-\bar{t})} W^{\mathrm{eq}, \lessgtr}(\omega),
$$

we obtain

$$
\mathcal{J}_{i k}^{\mathrm{ic}}(t)=i \sum_{n p} \mathcal{G}_{i n}^{R}(0, t) f_{\text {inp }}(t) \mathcal{G}_{p k}^{A}(0, t),
$$

where the tensor $f_{\text {inp }}(t)=\int \frac{d \omega}{2 \pi} e^{-i \omega t} f_{\text {inp }}(\omega)$ is the Fourier transform of

$$
f_{i n p}(\omega)=\sum_{m} W_{i m}^{\mathrm{eq},>}(\omega) \int_{-\infty}^{0} d \bar{t} e^{i \omega \bar{t}} \mathcal{G}_{n m}^{>}(0, \bar{t}) \mathcal{G}_{m p}^{<}(\bar{t}, 0) .
$$

The time integral in the expression above can be done analytically by using Eq. (25) and one finds

$$
f_{i n p}(\omega)=\frac{1}{i\left(\omega+\epsilon_{n}-\epsilon_{p}\right)+\eta} \sum_{m} \bar{\rho}_{n m}^{\mathrm{eq}} W_{i m}^{\mathrm{eq},>}(\omega) \rho_{m p}^{\mathrm{eq}} .
$$

The same considerations can be applied to the second term $\overline{\mathcal{J}}_{i k}^{\text {ic }}(t)$ of the IC integral in Eq. (16), yielding an expression for the IC integral suitable for numerical implementations. In fact, the tensor $f_{\text {inp }}(t)$ can be calculated separately for the needed time interval, and the computational effort scale linearly with the number of time steps. Furthermore, the IC integral in the $G W^{\mathrm{eq}}$ approximation scales with the fourth power of the number of basis functions $N_{b}$ (this should be compared with the $N_{b}^{5}$ scaling of IC integral in the $2 \mathrm{~B}$ approximation). Thus the $G W^{\text {eq }}$ approximation is numerically feasible.
[1] P. Danielewicz, Ann. Phys. (NY) 152, 239 (1984).

[2] G. Stefanucci and R. van Leeuwen, Nonequilibrium Many-Body Theory Quantum Systems: A Modern Introduction (Cambridge University Press, Cambridge, 2013).

[3] K. Balzer and M. Bonitz, Nonequilibrium Green's Functions Approach to Inhomogeneous Systems, Lecture Notes in Physics Vol. 867 (Springer, Berlin, Heidelberg, 2013).

[4] P. Lipavský, V. Špička, and B. Velický, Phys. Rev. B 34, 6933 (1986).

[5] E. Perfetto, A.-M. Uimonen, R. van Leeuwen, and G. Stefanucci, Phys. Rev. A 92, 033419 (2015).

[6] E. Perfetto, D. Sangalli, A. Marini, and G. Stefanucci, J. Phys. Chem. Lett. 9, 1353 (2018).

[7] G. Pal, Y. Pavlyukh, W. Hübner, and H. C. Schneider, Eur. Phys. J. B 79, 327 (2011).
[8] E. V. Boström, A. Mikkelsen, C. Verdozzi, E. Perfetto, and G Stefanucci, Nano Lett. 18, 785 (2018).

[9] D. Sangalli and A. Marini, EPL 110, 47004 (2015) [Errata: 117, 39901 (2017)].

[10] D. Sangalli, S. Dal Conte, C. Manzoni, G. Cerullo, and A. Marini, Phys. Rev. B 93, 195205 (2016).

[11] E. A. Pogna, M. Marsili, D. De Fazio, S. Dal Conte, C. Manzoni, D. Sangalli, D. Yoon, A. Lombardo, A. C. Ferrari, A Marini, G. Cerullo, and D. Prezzi, ACS Nano 10, 1182 (2016).

[12] A. Molina-Sánchez, D. Sangalli, L. Wirtz, and A. Marini, Nano Lett. 17, 4549 (2017).

[13] S. Hermanns, K. Balzer, and M. Bonitz, Phys. Scr. T151, 014036 (2012).

[14] S. Hermanns, K. Balzer, and M. Bonitz, J. Phys. Conf. Ser. 427, 012008 (2013). 
[15] S. Hermanns, N. Schlünzen, and M. Bonitz, Phys. Rev. B 90, 125111 (2014).

[16] S. Latini, E. Perfetto, A.-M. Uimonen, R. van Leeuwen, and G. Stefanucci, Phys. Rev. B 89, 075306 (2014).

[17] Y. Bar Lev and D. R. Reichman, Europhys. Lett. 113, 46001 (2016).

[18] H. Haug and A. Jauho, Quantum Kinetics in Transport and Optics of Semiconductors, Solid-State Sciences Vol. 123 (Springer, Berlin, Heidelberg, 2008).

[19] L. P. Kadanoff and G. Baym, Quantum Statistical Mechanics (Benjamin, New York, 1962).

[20] A. Stan, N. E. Dahlen, and R. van Leeuwen, J. Chem. Phys. 130, 224101 (2009).

[21] P. Myöhänen, A. Stan, G. Stefanucci, and R. van Leeuwen, Europhys. Lett. 84, 67001 (2008).
[22] P. Myöhänen, A. Stan, G. Stefanucci, and R. van Leeuwen, Phys. Rev. B 80, 115107 (2009).

[23] M. Puig von Friesen, C. Verdozzi, and C.-O. Almbladh, Phys. Rev. Lett. 103, 176404 (2009).

[24] M. Puig von Friesen, C. Verdozzi, and C.-O. Almbladh, Phys. Rev. B 82, 155108 (2010).

[25] H. Haug, Phys. Status Solidi 173, 139 (1992).

[26] M. Bonitz, D. Semkat, and H. Haug, Eur. Phys. J. B 9, 309 (1999).

[27] B. Arnaud, S. Lebègue, and M. Alouani, Phys. Rev. B 71, 035308 (2005).

[28] A. Marini, J. Phys. Conf. Ser. 427, 012003 (2013).

[29] G. Pal, Y. Pavlyukh, H. C. Schneider, and W. Hübner, Eur. Phys. J. B 70, 483 (2009).

[30] M. Hopjan, Ph.D. thesis, Lund University, 2018.

[31] E. Perfetto and G. Stefanucci (unpublished). 